\title{
Microstructural and Analytic Characterisation of Li-Ion Batteries by Correlative EDS, SIMS, Light-, Raman-, Ion- and Electron Microscopy
}

\author{
U. Golla-Schindler ${ }^{*}$, L. Han ${ }^{2}$, P. Gnauck ${ }^{2}$, F. Khanom² ${ }^{2}$ T. Bernthaler ${ }^{1}$ and G. Schneider ${ }^{1}$ \\ 1. Materials Research Institute (IMFAA), Aalen University, Aalen, Germany. \\ 2. Carl Zeiss Microscopy GmbH, Carl-Zeiss-Straße 22, Oberkochen, Germany. \\ * Corresponding author: ute.golla-schindler@hs-aalen.de
}

Li-ion batteries (LIBs) has become one of the most prevalent electrical storage systems due to its high volumetric and gravimetric energy density. Depending on the cell chemistry, design and operation several aging processes occur, which affect their performance and durability. Especially the detection of $\mathrm{Li}$ is one of the challenges for further understanding. The following investigations were performed on commercial available graphite/NMC $\left(\mathrm{Li}\left[\mathrm{Co}_{1 / 3} \mathrm{Ni}_{1 / 3} \mathrm{Mn}_{1 / 3}\right] \mathrm{O}_{2}\right)$ pouch cells, a reference $(\mathrm{R})$, a cycled $(\mathrm{C})$ and a storage (S) one [1]. Nondestructive Computer Tomography (CT) yields a thickness increment of the treated cells. Light microscopy (LM) on cross sections enables the macroscopic inspection. The morphology changes in the nm scale were studied with SEM, using a SIGMA 300 VP and a Crossbeam 540. Figure 1 shows SEM images of cathode and separator foils in top-view (a-d) and anode foils in cross-section (e, f). The cathode foils consist of NMC active material particles and conductive additives with binder material (Figure 1(a), marked as (1) and (2), respectively). The comparison of the reference with the aged films shows a surface coating on the aged NMC grains (Figure $1(\mathrm{~b})$, marked as $(3,4)$ ) also visible on the magnified inset of a single NMC particle. The detection of low-loss reflected backscattered electrons for energies beyond $3 \mathrm{keV}$ improves the available spatial resolution for material contrast. This offered the opportunity to address the average atomic number of the precipitates in the $\mathrm{nm}$ range inside the coating to be above carbon and below $\left(\mathrm{Li}\left[\mathrm{Co}_{1 / 3} \mathrm{Ni}_{1 / 3} \mathrm{Mn}_{1 / 3}\right] \mathrm{O}_{2}\right)$. On top of the separator foils, see Figure 1(c, d), spherical particles in the order of $10 \mathrm{~nm}$ to 1 micron $(5,6)$ and net-like structures (7) arise. In the cross-sectional image of the anode foils Figure 1(e, f), a reaction rim with a thickness of a few $\mu \mathrm{m}$ formed by aging is visible. Correlative measurements enable to combine macroscopic to nanoscopic and analytic information. In Figure 2(c) a top view LM image of a treated cell delivered bright and dark appearing areas, which were correlatively studied with SEM using ZEN 2 core. Figure $2(\mathrm{a}, \mathrm{b})$ were taken under the same instrument and imaging settings. The appearing significant brightness difference is caused by a change in the electronic conductivity (potential contrast). Figure 2(d) show correlative Raman-SEM applied on a treated anode foil. There the relative ratio of the $\mathrm{D}$ (characteristic for diamond, sp3 hybridization) and the $\mathrm{G}$ band (characteristic for sp2 hybridization graphite) shows differences. They are presented as a color-coded overlay on the SEM image. The red marked areas have an increased D band contribution, this indicating a rise of border areas or implemented lattice distortions in the graphite particles. Analytic studies by EDS of the precipitates on top of the separator foil delivered an enriched content of F, Si O, C [3], where the information on the most important element $\mathrm{Li}$ is missing. The ORION NanoFab (ZEISS) is an instrument based on helium and neon ion beams, newly equipped with a Secondary Ion Mass Spectrometer (SIMS) producing element maps with lateral resolution down to $15 \mathrm{~nm}$. This enables correlative SEM imaging and mapping of $\mathrm{Li}$ where all precipitates on top of the treated separator foil (big and small spherical particles marked as 1 and 2, respectively, and the flake-like features (3)) show an enriched Li content. Systematic study on morphology by CT, LM and SEM and the application of correlative LM-SEM, Raman-SEM and SEM-NanoFab SIMS delivered new unique insights in degeneration effects of Li-ion batteries [4]. 
References:

[1] M Bauer, J. Power Sources 317 (2016), p. 93.

[2] U Golla-Schindler et al., Micron 113 (2018), p. 10.

[3] U Golla-Schindler et al., EMAS (2019), accepted.

[4] We thank C Guenther and MA Danzer for the samples and Federal Ministry for Economic Affairs and Energy (BMWi) for financial support.

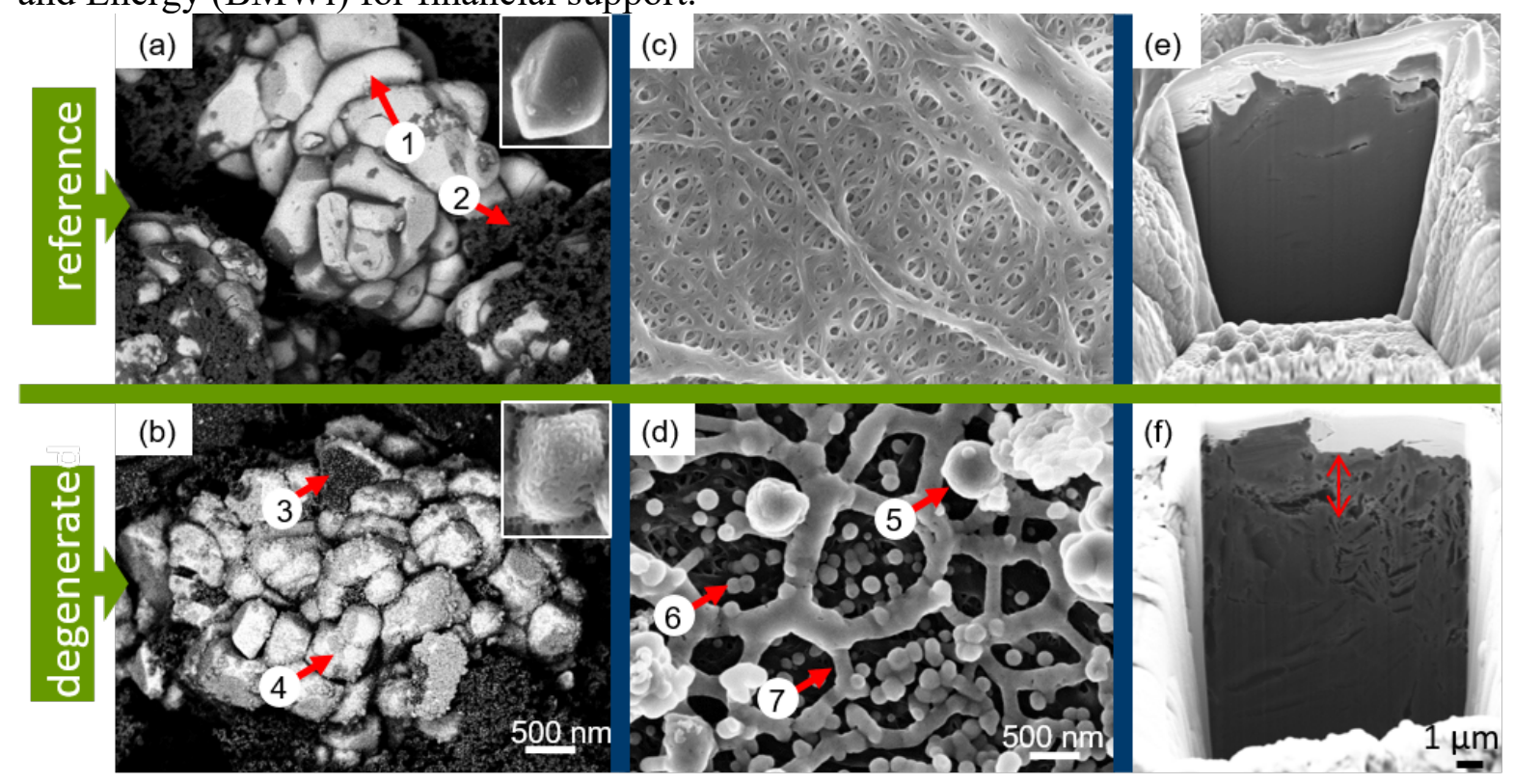

Figure 1. Morphology studies with SEM on the reference- $(\mathbf{a}, \mathbf{c}, \mathbf{e})$ and the treated- $(\mathbf{b}, \mathbf{d}, \mathbf{f})$ battery foils. SEM top view (a-b) cathode, (c-d) separator, (e-f) anode, cross sectional view obtained with the FIB.
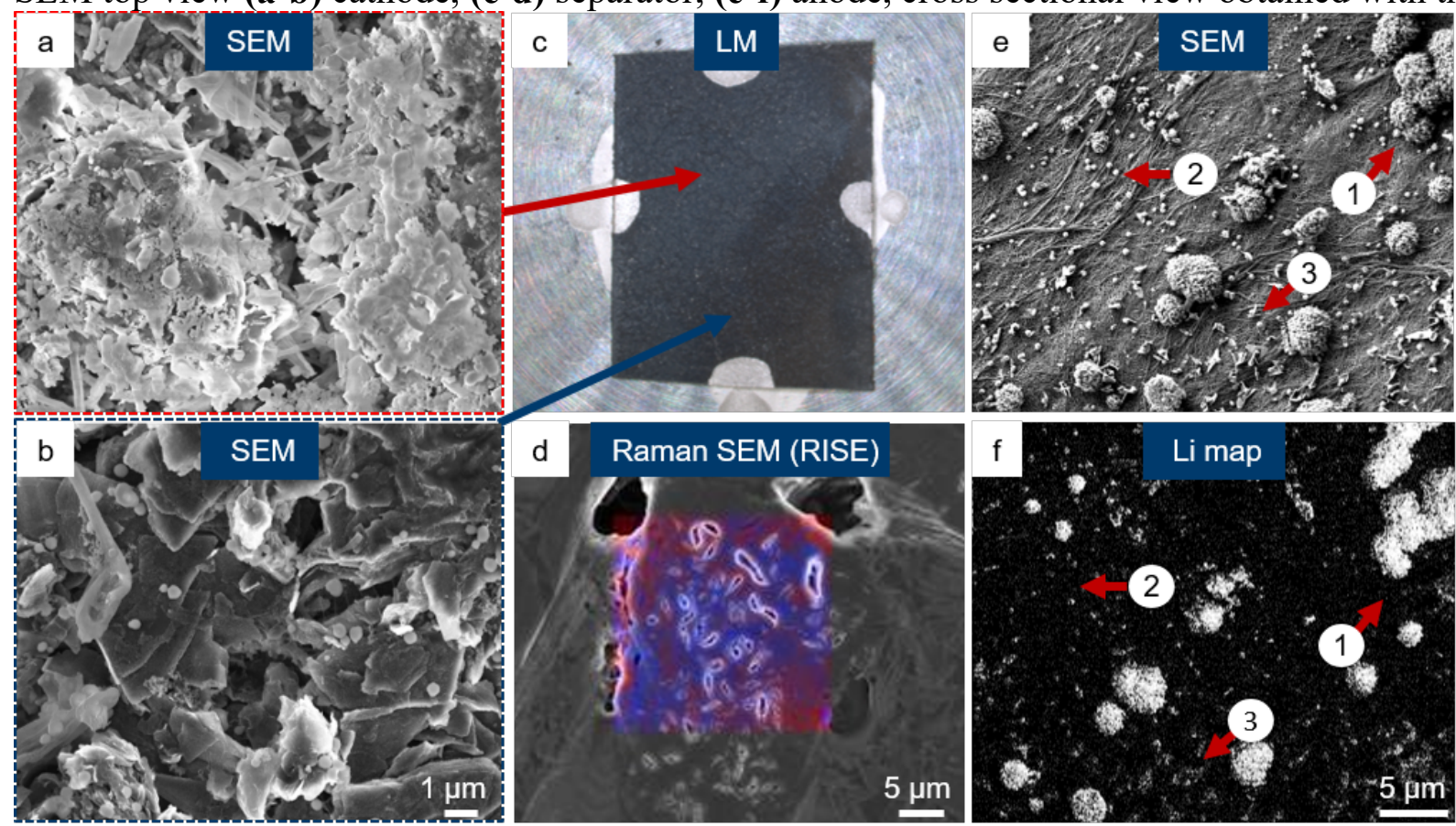

Figure 2. Correlative LM-SEM, Raman-SEM and SEM-Ne NanoFab SIMS, applied on Li-ion batteries. (a-c) Anode foil of a treated cell (a, b) SEM and (c) LM. (d) Raman SEM on a treated anode foil. (e, f) Correlative SEM NanoFab SIMS (e) SEM, (f) Li- map. 